\title{
XXIII.
}

\section{Friedrich Jodl,}

geb. 23. August 1849, gestorben 26. Januar 1914.

Von

Privatdozent Dr. Walther Schmied-Kowarzik in Wien.

Mit Friedrich Jodl hat die deutsche Gelehrtenwelt eine ihrer hervorragendsten und markantesten Persönlichkeiten verloren: einen Philosophen ebenso bedeutsam durch seine geschlossene Weltanschauung als durch seine ins Leben hinauswirkende, charaktervolle Gesinnung.

Jodls Weltanschauung kennzeichnet sich als Positivismus. Schon seine erste Schrift, die Doktordissertation vom Jahre 1871, war der Darstellung eines positivistischen Denkers gewidmet: David Hume's. Und seine letzte Buchveröffentlichung ist die Monographie iiber Ludwig Feuerbach (1904), dem Jodl näher steht, da er dem phänomenalistisch-immanenten Standpunkt Humes gegenüber den erkenntnistheoretischen Realismus vertritt. Jodl gehört demnach unter den Positivisten nicht zur Gruppe, die von Hume, Mill, Laas, Mach, Empiriokritizismus, Immanenzphilosophie gebildet wird, sondern zu der von Feuerbach, Comte, Spencer, Dühring, Riehl, Dilthey vertretenen Gruppe.

Wie Jodl allen theoretischen Idealismus und Phänomenalismus bekämpfte, um der Natur und den Naturwissenschaften gerecht zu werden, so lehnte er allen materialistischen Naturalismus ab, der „hilflos eïer ganzen Hemisphäre des Daseins abgewendet", das geistige Leben verkennt und vernachlässigt. Dieses Verständnis der Welt des Geistes und seiner Geschichte verknüpft ihn ebenso wie Feuerbach ,mit dem nachkantischen Idealismus und mit dem historischen Geiste der Romantik". 
In seiner zweibändigen „Geschichte der Ethik": (1882/89, 2. Aufl. 1906/12), einem standard work der philosophischen Literatur, bekundet Jodl diese „FFeinhörigkeit für die so unendlich mannigfaltigen Motive, welche in der Gedankensymphonie der Menschheit anklingen". Diese Geschichte ist zugleich eine psychologisch-analytische Behandlung der sittlichen Tatsachen und verweist so auf das zweite Hauptwerk Jodls, das "Lehrbuch der Psychologie" (1897, 2. Aufl. 1903, 3. Aufl. 1908, 4. Aufl. 1914). Die Psychologie ist nach Jodl die Grundwissenschaft der Philosophie, freilich nicht die einseitig experimentelle, und damit auf einen Ausschnitt des Bewußtseins beschränkte, sondern seine empirisch-analytische Psychologie, die der Totalität des Seelenlebens gerecht wird, neben der Rezeptivität die Spontaneität anerkennt und in einem reichen System feinst abgestufter Unterscheidungen die Mannigfaltigkeit der Erlebnisse harmonisch erfaßt.

In beide Werke ist die gesamte Literatur der Deutschen, AngloAmerikaner, Franzosen und Italiener hineingearbeitet, so daß in Gelehrtenkreisen das. Wort im Umlauf ist: Jodls Psychologie sei selbst ein psychologisches Phänomen. Diesem umfassenden Inhalt entspricht die vollendete Form. Jodl ist einer der ,ausgezeichnetsten Prosaschriftsteller des deutschen Volkes", dem (wie Hugo Spitzer sagte) „nur sehr wenige von Lessing bis Nietzsche und Eucken gleichkonmen".

Die gleiche Formvollendung zeigen seine Reden und Vorträge, deren berühmtesten er in der „Deutschen Gesellschaft für ethische Kultur" und im Wiener Volksbildungsverein gehalten. Praktische Menschenliebe war es, die ihn in beiden Organisationen für eine vertiefte Geistesbildung des Volkes wirken ließ, und seine Worte erweckten in aller Herzen echte Begeisterung und werkbereite Gesinnung, denn sie waren getragen von jenem ethischen Idealismus, von dem seine ganze Persönlichkeit durchdrungen war.

So erscheint uns Friedrich Jodls Wesen voll und ganz ausgesprochen in dem schlichten, tiefgefühlten Wort der hochsinnigen Frau, die ein Dritteljahrhundert seine Lebensgefährtin war: „Ein großer und reiner Mensch schied aus dem Leben."

Sein Andenken wird allen, die von seiner Persönlichkeit berührt wurden, teuer sein! 
Im Nachfolgenden sind Triedrich Jodls Schriften verzeichnet. Nach seinem Tod erschien der Aufsatz über Gerhart Hauptmanns Festspiel und die kleine Schrift „Vom wahren und falschen Idealismus". Die Sammlung seiner Aufsätze und Vorträge wurde von Jodl selbst vorbereitet und geordnet und wird in zwei Bänden im Laufe des Jahres auf den Büchermarkt kommen. Die nachgelassene erkenntnistheoretische Schrift „Kritik des Idealismus", die Jodl selbst als sein drittes Hauptwerk bezeichnete, wird voraussichtlich Anfang 1915 erscheinen.

Eine Monographie von Wilhelm Börner (Stuttgart, bei Cotta, 1911) bringt Jodls gesamte Lebensarbeit in größter Objektivität (meist in wörtlichen Zitaten) zur Darstellung.

Verzeichnis der Veröffentlichungen Friedrich Jodls*) (von ihm selbst zusammengestellt; überarbeitet von W. Schmied-Kowarzik).

[Die wichtigsten Schriften sind gesperrt; $\mathrm{B}=$ Besprechung; $\mathrm{V}=$ Vortrag; A. Z. = Allg. Zeitung, Augsburg; Ph. M. = Philosoph. Monatshefte; S. Pr. = Süddeutsche Presse, München; DLZ. = Deutsche Literatur-Zeitung; Z. f. Ph: = Zeitschrift für Philosophie und philosophische Kritik; A.f. Ph. = Archiv für systematische Philosophie; E. K. = Ethische Kultur; MGEK. = Mitteilungen der Deutschen Gesellschaft für ethische Kultur; MEGW. $=$ Mitteilungen der ethischen Gesellschaft in Wien; MWV. = Mitteilungen des Wiener Volksbildungsvereins; Ö. R. = Österreichische Rundschau, Wien; N. fr. Pr. = Neue freie Presse, Wien; I. j. e. = International journal of ethics.]

1871

1. David Humes Lehre von der Erkenntnis. Halle bei Pfeffer. (Dissertation.)

1872

2. Leben und Philosophie David Humes. Halle bei Pfeffer (jetzt bei Alfred Kröner, -Leipzig). (Von der Universität zu München 1870 gekrönte Preisschrift.)

1874

3.B Edm. Pfleiderer, „Empirismus und Skepsis in Humes Philosophie“. (A. Z. 13. Okt.)

4.B Whitney-Jolly, „Die Sprachwissenschaften“. (A. Z. 8. Dez.)

*) Die mit einem Sternchen $\left(^{*}\right)$ bezeichneten Schriften sind in der oben erwähnten Sammlung, die den Titel „Vom Lcbenswege“ führt und im Herbste bei J. G. Cotta (Stuttgart) erscheinen wird, enthalten. 
5. Naturwissenschaft und Philosophie (Gid. Spicker). (A. Z. 8. Jan.)

1877

6.B Caro, „Problèmes de morale sociale“. (Ph. M. Bd. 13 S. 286.)

7.B G. Mehring, „Die philosophisch-kritischen Grundsätze der Selbstvollendung und die Geschichtsphilosophie". (Ph. M. Bd. 13 S. 393.)

8. Zur Philosophie der Geschichte (Laurent, Flint, Meyr). (A. Z. Nr. 117; Ph. M. Bd. 14 S. 112.)

\section{8}

9. Die Kulturgeschichtsschreibung. Halle bei Pfeffer (jetzt bei Alfred Kröner, Leipzig).

10.B Pfenninger, „Der Begriff der Strafe“. (Ph. M. Bd. 14 S. 170.)

11.B Paul Lilienfeld, „Gedanken über die Sozialwissenschaften der Zukunft", I., II., III. T. (Ph. M. Bd. 14 S. 491.)

12. Die Philosophie der Geschichte (Rocholl). (A. Z. Nr. 275-77.)

13. Über das Verhältnis zwischen Philosophie und Mode. (Wartburg V, Nr. 5 u. 6.)

\section{9}

*14. Nekrolog auf Johannes Huber. (S. Pr. 22. März; Ph. M. Bd. 15 S. 319.)

*15. J. Hubers Stellung in der deutschen Philosophie. (Deutsche Revue J. 3 H. 11.)

16.B Alfred Fouillée, „L'idée moderne du droit“. (Ph. M. Bd. 15 S. 175.)

1880

17. Studien zur Geschichte und Kritik der Theorien über den Ursprung des Sittlichen (Hobbes und seine Gegner im 17. Jahrhundert). (Als Habilitationsschrift gedruckt.)

18.B P. v. Lilienfeld, „Gedanken über die Sozialwissenschaften der Zukunft“, IV. T. (Ph. M. Bd. 16 S. $549 f$.)

19. Bildung und Halbbildung. (S. Pr. 26. Okt.)

1881

20. Die kulturgeschichtliche Literatur der letzten 10 Jahre. (Meyers Konversationslexikon, Jahressupplement 1880/81.)

21. Bayerische Literaturbriefe, I. (S. Pr. 15. Mai.)

*22. Johannes Huber, ein kritischer Rückblick (Zirngiebl). (S. Pr. 24., 25. Aug., Nr. 197/98.)

23. Das Monstrekonzert. (Festzeitung f. d. 7. deutsche Bundesschießen.)

- 24.B Alf. Fouillée, „La science sociale contemporaine“. (Ph. M.Bd. 17 S. 266.)

\section{2}

25. Geschichte der Ethik. Bd.I. Stuttgart bei Cotta.

26.B Ed. Zirngiebl, „Joh. Huber“. (Ph. M. Bd. 18 S. 170.)

27.B Hayem, „L'être sociale“. (Ibid. S. 370.) 
1883

28.B Carl Peters, „Willenswelt und Weltwille“. (DLZ. Nr. 15.)

29.B Hugo Sommer, „Der Pessimismus und die Sittenlehre“; ders., „Das Wesen und die Bedeutung der menschlichen Freiheit". (Göttg. Gel. Anz. Nr. 11 u. 12.)

30.B Gutberlet, „Ethik und Naturrecht“. (DLZ. Nr. 43.)

31.B Kunstausstellungsberichte v. Philalethes. (Wartburg.)

32.B Sallwürk und Vogt, „Rousseau's Emile“. (DLZ. Nr. 50.)

\section{4}

33.B Lazarus, „Die Reize des Spiels“. (DLZ. Nr.13.)

34.B Fouillée, „Critique des systèmes de morale contemporaine. (Ph. M. Bd. 20 S. 549.)

35.B K. A. Schmid, „Geschichte der Erziehung“. Bd. I. (Cottasche Zeitschs f. allg. Gesch. H. 8.)

36. Über neuere kulturgeschichtliche Literatur. (Meyers Konversationslexikon, Jahressupplement.).

\section{5}

37. Der Nativismus und die bayr. Universitäten. (N. Nachr. Nr. 46, 47, 64.)

38. Bildungsfragen. (N. Nachr. Nr. 215.)

39.B Al. Meinong, „Philos. Wissenschaft und ihre Propädeutik“". (DLZ. Nr.31.) 40.B Salter, „Die Religion der Moral“‘ (DLZ. Nr. 45.)

1886

*41. Volkswirtschaftslehre und Ethik. (Deutsche Zeit- und Streitfragen Nr. 224.) Berlin bei C. Habel.

42.B Christaller, „Die Aristokratie des Geistes“. (Ph. M. Bd. 22 S. 267.)

43.B Fouillée, „La propriété sociale et la democratie“.. (Ph. M. Bd. 22 S. 281.)

44.B Noah Porter, „Elements of moral science“. (Ph. M. Bd. 22 S. 618.)

*45.B A. Spir, ,,Schriften zur Moralphilosophie“, und Rud. Kittel, , ,Sittliche Fragen". (Z. f. Ph. Bd. 88 S. 291.)

46.B Salter, „Die Religion der Moral“, und Cathrein, „Die Sittenlehre des Darwinismus" (Z. f. Ph. Bd. 89 S. 135:)

47.B Romundt, „Die Vollendung des Sokrates“.. (Ibid. Bd. 89 S. 129.)

48.B L. Buro, „Die Begründung der sittl. Gesetze vom Standpunkt der natürl. Erkenntnis", und Emil Kater, „Die Ethik des Utilitarismus". (DLZ. Nr. 9.)

49.B. Herm. Steinthal, „Allg. Ethik“. (DLZ. Nr. 14.)

50.B Rich. Falckenberg, „Geschichte der Philosophie“ (Ibid.' Nr. 26.)

51.B Class, "„Ideale und Güter“. (Ibid. Nr. 36.)

1887

52.B Hans Voltz, „Die Ethik als Wissenschaft". (DLZ. Nr. 20.)

53.B Rich. Wallaschek, „Ideen z. prakt. Philosophie“. (DLZ. Nr. 49. Z. f. Ph. 1888 Bd. 92 S. 154.) 
54.B Fr. J. Mach, „Die Willensfreiheit des Menschen“, und Thoden von Velzen, „Über die Geistesfreiheit vulgo Willensfreiheit". (DLZ. Nr. 27.)

55.B Köstlin, „,Geschichte der Ethik“‘. Bd. I. (DLZ. Nr. 48; Z. f. Ph. 1889 Bd. 96 S. 292.)

56.B Paul Rée, „Die Illusion der Willensfreiheit“. (Z.f. Ph. Bd.90 S. 300.)

57.B O. Flügel, „Das Ich und die sittlichen Ideen“. (Z. £. Ph. Bd. 91 S. 142.)

58.B C. N. Stracke, „Ludwig Feuerbach“. (Z. f. Ph. Bd. 91 S. 300.)

59.B Wundt, „Ethik“. (Ph. M. S. 66.)

60.B Carneri, „Entwicklung und Glückseligkeit. Ethische Essays“. (Ph. M.)

61B. Sigwart, „,Vorfragen der Ethik“. (DLZ. Nr.37; Ph. M. 1888 Bd. 25 H. VI.)

\section{8}

"62.B A. Spir, „Vermischte Schriften“. (Z. f. Ph. Bd. 91 S. 156.)

63.B Lehmann, „Das Problem der Killensfreiheit", und Duskowitz, „Verantwortung und Zurechnung"6. (Z. f. Ph. Bd. 93 S. 308.)

64. B Ac. Franck, ,Phiosophie du droit civil“‘. (Ph. M. BJ. 25 S. 90.)

65.B Franz Staudinger, „Die Gesetze der Freiheit“. (DLZ. Nr. 12; Ph. M. Bd. 25 S. 85.)

66.B C. Hebler, „Elemente einer philos. Freiheitslehre“, und Otto Lehmann, „Das Problem der Willensfreiheit“". (DLZ. Nr. 1.)

67.B Waldemar Meyer, „Die Wahlfreiheit des Willens in ihrer Nichtigkeit dargelegt". (DLZ. Nr. 7.)

68.B Cesca, „L'educazione del carattere“: (DLZ. Nr. 31.)

\section{9}

69B. Geschichte der Ethik. Bd. II. Stuttgart bei Cotta.

70.B Döring, „Philosophische Güterlehre“. (DLZ. Ṅr. 4; Ph. M. 1890 Bd. 26 S. 441 H. V-VI.)

71.B Küßner, „Kritik des Pessimismus“: (DLZ. Nr. 4.)

72.B Gizycki, „Moralphilosophie“, und Paulsen, „System der Ethik“. (DLZ. Nr. 21; Ph. M. 1890 Bd. 26 S. 210.)

73.B Scherejew, „Selbstsein“, und P. Hensel, „Ethisches Wesen und ethisches Handeln". (DLZ. Nr. 28.)

74.B Alexander; „Moral Order and Progress“. (DLZ. Nr. 40.)

75.B Steinitzer, „Die menschlichen und tierischen Gemütsbewegungen“. (DLZ. Nr. 44.)

76B. Wollug, „Leitfaden der Moral“, „Über die Grenzen menschlicher Erkenntnis". (Z. f. Ph. Bd. 96 S. 297.)

*77.B Wilbrandts „Meister von Palmyra“. (Voss. Ztg. Nr. 24, 16. Juni.)

.78. Giordano Bruno. (Wissenschaftl. Rundsch. d. M. N. Nachr. Nr. 300 und 302.)

1890

*79. Schelling. (Allg. deutsche Biographie, Lpzg.)

80.B Höffding, „Einleitung in die englische Philosophie unserer Zeit", und Ravaisson, „Die französ. Philosophie des 19. Jahrhunderts'. . (DLZ. Nr. 1.) 
81.B Höffding, „Ethik". (DLZ. Nr. 9.)

82.B Gruber, "A. Comte, der Begründer des Positivismus". (DLZ, Nr. 22.)

83.13 Pierre Laffitte, "Cours de philosophie premiere", Bd. I. (Ibid. Nr. 28.)

84.B Ziegler, „Sittliches Scin und sittliches Werden", und Kater, „Die Moral der. Zukunft". (DLZ. Nr. 33; Z. f. Ph. 1893 Bd. 101 S. 290.)

85.B Salter, „Moralische Reden", und Coit, „Die ethische Bewegung in der Religion". (DLZ. Nr. 40.)

86.B Eduard Fechtner, „Die praktische Philosophie und ihre Bedeutung für die Rechtsstudien“. (Ph. M. Bd. 26 S. 228.)

87.B Otto Caspari, „Drei Essays über Grund- und Lebensfragen der philosophischen Wissenschaft". (Z. f. Ph. Bd. 97 S. 294.)

\section{1}

88.B Harms, ,Ethik“, (Ph. M. Bd. 27 S. 188.)

89.B Wohlwill, „J. Jungius“. (Z. f. Ph. Bd. 98 S. 252.)

90.B Hoppe, „Der psychologische Ursprung des Rechts“. (Ibid. S. 282.)

91.B E. Laas, „Literarischer Nachlaß“, herausg. v. Kerry. (Ibid. S. 251.)

*92. Die Philosophie Robert Hamerlings. (Wissenschaftl. Rundsch. der M. N. Nachr. Nr. 115.)

*93. Grenzen des seelischen Lebens [Binet und Romanes]. (Wissenschaftl. Rundsch. d. M. N. Nachr. Nr. 519.)

94. German philosophy in the 19. century. (Monist, Vol. I Nr. 2, Jan.)

*95. Morals in history. (I. j. e. Vol. I Nr. 2, Jan.)

96.B La revue occidentale, hg. v. Pierre Laffitte. (DLZ. Nr. 5.)

97.B E. Große, „Herb. Spencers Lehre von dem Unerkennbaren“. (DLZ. Nr. 14.)

98.B V. Cathrein S. J., „MัMoralphilosophie“, Bd. I. (DLZ. Nr. 24.)

99.B R. Fester, „Rousseau und die deutsche Geschichtsphilosophịe“. (DLZ. Nr. 41.)

100.B Bolin, „Feuerbach“. (DLZ. Nr. 47; Ph. M. [1892] VIII.)

101. Jahresbericht über Erscheinungen der anglo-amerikanischen Literatur aus den Jahren 1888/89. (Z. f. Ph. Bd. 99 S. 157.)

\section{2}

*102. Moral, Religion und Schule. Stuttgart bei Cotta.

103.B Simmel, „Über soziale Differenzierung usw.", III. (DLZ. Nr. 4.)

104B. Elissen, „Fr. Alb. Lange“. (DLZ. Nr. 12.)

*105.B Hasbach, „Die allgem. philos. Grundlagen der politischen Ökonomie“. (DLZ. Nr. 14.)

106.B Mart. Keibel, „Die Religion und ihr Recht gegenüber dem modernen Moralismus". (DLZ. Nr. 16; MGEK.)

107.B Gruber, „Der Positivismus seit dem Tode Comtes“. (Ibid.)

108.B Roberty, „La philosophie du siècle“. (DLZ. Nr. 25.)

109.B Simmel, „Einleitung in die Moralwissenschaft", I. Bd. (DLZ. Nr. 29.; I. j. e. Vol. III, Okt.)

110.B F. M. Huber, „Dogmenlose Sittenlehre“. (DLZ. Nr. 44.) 
111.B L. Gumplovicz, „Soziologie und Politik“. (DIZ. Nr. 46.)

112.B Bernhard Duhm, „Kosmologie und Religion". (DLZ. Nr. 49.)

113.B Giov. Cesca, „Dell" Hobbes", „Il fattori dell' evoluzione filosofica usw.“. (DLZ. Nr. 51.)

114.B Thomas, „Principes de philos. morale usw.“. (Ph. M. IV S. 185.)

115.B J. Loewe, „Die spekulative Idee der Freiheit“. (Ph. M. IV S. 344.)

*116. Die ethischen Voraussetzungen von Krieg und Frieden. („Die Waffen nieder" Nr. 9.)

117.B J. Baumann, „Ein Lebensbund“. (I. j. e. Vol. III, Okt.)

118. 2. Jahresbericht über Erscheinungen der anglo-amerikanischen Literatur aus den Jahren 1890/91. (Z. f. Ph. Bd. 101 S. 87.)

1893

*119. Über das Wesen des Naturrechts und seine Bedeutung in der Gegenwart [V Wien 1893]. ' (Prager Jurist. Viertelj.-Schrift Bd. 25 H. 1.)

*120. Wesen und Ziele der ethischen Bewegung in Deutschland. [V Frankfurt und Prag]. (Frankfurt 1893, 4. Aufl. 1908.)

*121. Personleben und Gemeinschaftsleben [V Berlin]. (MGEK. Jahrg. 1 Nr. 2.)

122.B Duboc, "Grundriß einer einheitlichen Trieblehre". (Ph. M. S. 330.)

123.B Spicker, „Über die Ursachen des Verfalls der Philosophie“. (Ph. M. S. 458.)

124.B Helene Lange, „Vorträge und Schriften über Frauenfrage und weibliche Bildung". (I. j. e. Vol. III, 2.)

125.B Runze, „Ethik“. (Ibid. Vol. III, 2.)

126. Was heißt ethische Kultur? (E. K. Jahrg. I Nr. 1.)

127. Zur Schulfrage. Entgegnung an Natorp. (E. K. Nr. 14.)

128.B Ant. H. Rop [Pseudonym: Dr. Taussig], „Religionsunterricht und Erziehung". (E. K. Nr. 16.)

129. Religion oder Moralunterricht. Entgegnung an Schwerin. (Ibid. Nr. 17 und 20.)

130. Zur Erinnerung an Karl Märker. (E. K. Nr. 25.)

131.B Spindler, „Über die ethischen Wirkungen des Nationalgefühls“. (E.K. Nr. 31.)

*132. Selbstpflicht und Nächstenliebe. (E. K. Nr. 32.)

133. Einiges Christentum und ethische Kultur. (E. K. Nr. 39.)

134. Die ethische Bervegung in Deutschland. (N. Fr. Pr. 23. u. 25. Aug.)

135.B Andresen, „Die Entwicklung des.Menschen“, Robert, „Zur Herrschaft der Seele“, Henop, „Das Vergängliche, das Bleibende, das Ewige“, Weiß, „Die ethische Aufgabe des Menschen". (DLZ. Nr. 1.)

136.B Zerbst, „Nein und Ja.. Antwort an H. D. Türck". (DLZ. Nr. 6.)

137.B Josiah Royce, ,The spirit of modern philosophy“. (DLZ. Nr. 10.)

138.B Kennedy, „Gottesglaube und moderne Weltanschauung“, Katz, „Das Weltproblem und seine Lösung،‘. (DLZ. Nr. 13.)

139. Spencer, „The Principles of Ethics“, Vol. I. (DLZ. Nr. 15.)

140.B Friso, "Filosofia morale". (DLZ. Nr. 21.) 
141.J3 Guetano Negri, "Segni del Tempo". (DLZ. Nr. 25.)

142.B Oclzelt-Newin, „Uber sittliche Dispositionen. (DLZ. Nr. 27.)

143.B Sir Herbert Maxwell, „Meridiana: Noontide Essays". (DLZ. Nr. 30.)

144.B Chadwick, „Religion ohne Dogma“. (DLZ. Nr. 35.)

145.B Graue, „Dio selbständige Stellung der Sittlichkeit zur Religion“, Traub, „Dic sittliche Weltordnung“, Stange, „Die christliche Ethik in ihrem Verhältnis zur modernen Ethik". (DLZ. Nr. 46.)

146.B Jentsch, „Geschichtsphilosophische Gedanken“. (DLZ. Nr. 48.)

*147. Wissenschaft und moderne Theologie. (Monist Vol. III Nr. 3.)

148.B Spencer, „Religion and modern Science“. Ibid.

\section{4}

*149. Was heißt ethische Kultur. (Sammlung gemeinnütziger Vorträge. Prag, Nr. 191.)

150.B Herkner, „Die Zukunft der deutschen Österreicher“. (E. K. Nr.6.)

151.B Carneri, „Der moderme Mensch“. (E. K. Nr. 50.)

152. Zur Abwehr und Verständigung. (E. K. Nr. 5l.)

153. Ethische Kultur und soziale Organisation. (E. K. Nr. 14, 15, 18; MGEK. II, 2.)

154.B Bösch, „Das Mitgefühl". (Ph. M. Bd. 30 H. III u. IV S. 104.)

155.B Wundt, „Ethik“, 2. Aufl. (Ph. M. Bd. 30 H. III u. IV S. 205.)

156.B Hedwig Bender, „Das Wesen der Sittlichkeit“. (Ph. M. Bd. 30 H.V u. VI S. 320.)

157. Einleitung zu „Träume" von Olive Schrẹiner, übersetzt von meiner. Frau. (Berlin bei F. Dümmler.)

*158. Jahresbericht über Erscheinungen der anglo-amerikanischen Literatur a. d. Zeit von 1891/92. (Z. f. Ph. Bd. 104.)

*159. Spinoza. Auf Grund einer neuen Darstellung. (Die Nation Nr. 9 S. 122.)

160. Das Preisausschreiben der deutschen Gesellschaft f: ethische Kultur (Die Nation Nr. 37; MGEK. II, 4.)

161.B Zeitgenoß, „Moralische Träumereien“, Heims, „Lebensfragen“. (DLZ. Nr. 3.)

162.B J. B. Meyer, „Der Mainzer Katholikentag, der Fall Harnack und die Gottlosigkeit unserer Universitäten". (DLZ. Nr. 1.)

163.B Cadorna, „Religione, Diritto, Libertà22. (DLLZ. Nr. 8.)

164.B Wilh. Weigand, „Essays“, Weigand, „Fr. Nietzsche“. (DLZ. Nr. 20.)

165.B Glogau, „Tolstoi", Saitschik, „Dostojewski und Tolstoi“. (DLZ. Nr. 25.)

166.B Joyau, „La philosophie pendant la revolution“. (DLZ. Nr. 28.)

167.B Segall-Socolin, „Die Verjüngung der Philosophie“. (DLZ. Nr. 31.)

168.B Simmel, „Einleitung in die Moralwissenschaft“, Bd. II. (DLZ. Nr. 34.)

169.B Bolin, „Spinoza“. (DLZ. Nr. 36; Die Nation Jahrg. 12 Nr. 9.)

170.B Güttler, „Wissen und Glauben“. (DLZ. Nr. 39.)

171.B Nordau, „Entartung“. (DLZ. Nr. 44.)

172.B Frhr. v. Hertling, „Naturrecht und Sozialpolitik“, Reinhard Frank, „Naturrecht, geschichtliches Recht und soziales Recht“. (DLZ. Nr. 47.) 
1895

173. Ethik. Geschichtlicher Abriß bis zur Gegenwart. (In Reins „Enzyklop. Handbuch .d. Pädagogik"; Sonderdruck 1896; Langensalza bei H. Beyer.)

174. Volkswirtschaftslehre und Ethik. (Ins Russische übersetzt von Alex. Ostrogosky, Petersburg.)

175. Jahresbericht über Erscheinungen der Ethik aus dem Jahre 1894. (A. f. Ph. Bd. I, 4.)

*176. Über dasWesen und die Aufgabe der Ethischen Gesellschaft. (MEGW. 1; 4. Aufl. 1914.)

177. Verzeichnis gemeinverständlicher Schriften zur Einführung in die ethische Bewegung. (MEGW. 2.)

178. Die ethische Bewegung und die Religion. (E. K. Nr. 19 u. 20.)

179. Die ethische Bewegung und die öffentliche Meinung. (E. K. Nr. 40 und 41.)

*180. Georg v. Gizycki. Ein philosophisches Charakterbild. (Die Nation Jahrg. 12 Nr. 26.)

181. Litterary Correspondence Germany and Austria. Nr. I. (Monist, Vol. VI, Okt., 1.)

182. Philosophischer Briefwechsel über den Begriff des Dings an sich mit P. Carus. (The Metaphysical X in Cognition, Monist, Juli.)

*183. Neuere Systembildungen in der deutschen Philosophie. (Aula Jahrg. I, 17, 18.)

184. G. v. Gizycki und die Wissenschaft der Ethik. (I. j. e. Vol. V Nr. 4, Juli.)

185.B C. Gutberlet, „Ethik und Religion“. (DLZ. Nr. 5.)

186.B Dessoir, „Geschichte der neueren deutschen Psychologie“, Bd. I. (DLZ. Nr. 10.)

187.B Gustav Engel, „Versuch einer ontologischen Begründung des Seinsollenden. (DLZ. Nr. 11.)

188.B Vignoli, „Peregrinazioni Psicologiche. (DLZ. Nr. 17.)

189.B Leopold Besses, „Das der Menschheit Gemeinsame“, M. R. v. Stern, „Stimmen der Stille", Séverin Icard, „Paradoxes et verités". (DLZ. Nr. 31.)

190.B Tolstoi, „Religion und Moral“. (DLZ. Nr. 32.)

191.B Anonymus, „Die Sittlichkeitslehre als Naturlehre". (DLZ. Nr. 32.)

192.B Kuhlenbeck, „Giordano Brunos Dialog vom Unendlichen, dem Universum und den Welten". (DLZ. Nr. 39.)

193.B Giov. Cesca, „Contributo alla storia del fenomenismo“, idem, „L'idealismo soggetivo di J. G. Fichte“. (DLZ. Nr. 41.)

194.B Bregenzer, „Thier-Ethik“. (DLZ. Nr. 44.)

195.B Annie Besant, „Reincarnation“, A. Lillie, „Modern Mystic and Modern Magic". (DLZ. Nr. 51.) 
196. Lehrbuch der Psychologie. Stuttgart bei Cotta. (Am Titelblatt: 1897.)

197. Jahresbericht über Erscheinungen der anglo-amerikanischen Jiteratur i. J. 1893. (Z. f. Ph. Bd. 107 S. 103.)

*198. Die Prometheussage und ihre ethische Bedeutung. (E. K. Jahrg. 4 $\mathrm{Nr} .5-\mathrm{S}$.

199. Musik und Metaphysik. (Die Zukunft, 30. Mai, Nr. 35.)

*200. On the Origin and Import of the Idea of Cansality. (Monist Vol. II Nr. 4 S. 516.)

1897

201. Grillparzer und die Philosophie. Bruchstücke aus einem in der Grillparzer-Gesellschaft gehaltenen Vortrag. (Wiener Zeitung Nr. 1, 2.)

*202. Goethes Stellung zum religiösen Problem. (Auszug aus dem gleichnamigen Vortrag, Chronik des Wiener Goethe-Vereins, Bd. XI Nr. 7 und 8; vgl. Die Wage [1898] Nr. 31 u. 32.)

203. James Seth, „A Study of Ethical Principles“. (DLZ. Nr. 1.)

204.B Toland, „Das Pantheistikon“. Übersetzt von Fensch. (DLZ. Nr. 28.)

*205. Hundert Jahre nach Kant. Anknüpfend an Kronenbergs Kant. (N. fr.Pr. 15. Jan.)

*206. Die Moral in der Weltgeschichte. Ein philosophisches Gespräch. (E. K. Nr. 20 u. 21.)

\section{8}

207. Jahresbericht über die Erscheinungen der Ethik aus dem Jahre 1895. (A. f. Ph. Bd. IV, 3.)

*208. Grillparzer und die Philosophie. (Jahrbuch d. Grillparzer-Gesellsch. Jahrg. 8.)

209. Grillparzers ästhetische Anschauungen. (Wiener Ztg., Dez.)

210. Ludwig Feuerbach. (Das 19. Jahrhundert in Bildnissen. Berlin, Verlag d. photograph. Gesellschaft.)

*211. D. Fr. Strauß in seinen Briefen, herausg. von E. Zeller. (Euphorion Bd. V H. 2 S. 318.)

*212. J. G. Fichte als Sozialpolitiker. (E. K. Nr. 10 u. 11; Z. f. Ph. Bd. 13.)

*213. Nekrolog auf Rob. Zimmermann. (Bericht über den Rektoratswechsel 1898/99.)

214. Bericht über die Philosophie an der Wiener Universität in der Zeit zwischen 1848 und 1898. (Jubiläumsfestschrift d. Wiener Universität. 2. Dez.)

*215. Was leistet das humanistische Gymnasium für die allgemeine Bildung? (In den Protokollen d. Mittelschul-Enquête der "Wage“.)

216.B A. Faggi, Principii di psicologia moderna. (DLZ. Nr. 1.)

\section{9}

217. Litterary Correspondence. (Monist Vol. IX, 2.)

218B. Ein Handbuch der menschlich-natürlichen Sittenlehre [A. Döring]. (E. K. Nr. 6 u. 7.) 
219. R. Zimmermanns wissenschaftliche Bedeutung. (Bericht üb. d. phil.hist. Kl. d. k. Akademie d. W.)

220. Adresse der Wiener Professoren an Czar Nikolaus. (In: Pro finnlandia.)

221. F. E. Beneke. (D. 19. Jh. in Bildnissen.)

222. Höhere Mädchenbildung und die Gymnasialfrage. (Dokumente der Frauen Bd. I Nr. 6.)

223. Das 19. Jahrhundert. Eine Sozialbetrachtung. (Fremdenblatt, 31. Dez.) 1900

224. Jahresbericht über die Literatur und Ethik aus den Jahren 1895/96. (A. f. Ph. Bd. VI, 2.)

*225. Goethe als Bildungsträger. Festrede zur Feier seines 150. Geburtstages, gehalten in Brünn. (Jahresbericht des deutschen Hauses; Auszug: Wiener Ztg. Nr. 18 u. 19.)

*226. Grillparzers Ideen zur Ästhetik. (Jahrb. d. Grillp.-Gesellsch.)

227.B Th. Ziegler, „Die geistigen und sozialen Strömungen des 19. Jahrhunderts." (Euphorion Bd. II H. 4.)

*228. Goethe und Kant. Festgabe zu N. Dumbas 70. Geb. (Monist, Jan.1901, engl.; Z. f. Ph. [1902] Bd. 120.)

229. Giordano Bruno. (N. fr. Pr., 18. Feb.)

230. Zur Einführung in die Philosophie. (N. fr. Pr., 6. Mai.)

*231. Altes und Neues über Schopenhauer. (N. fr. Pr., 7. Okt.)

232. Ein österreichischer Philosoph. B. v. Carneri zum 80. Geb. (N. fr. Pr., 3. Nov.)

*234. Leo Tolstoj.

235. H. Ths. Buckle.

236. J. Bentham.

237. Henri George.

238. Ludwig Büchner.

239. Saint-Simon.

240. Huldigungs-Adresse der deutschen Universitäten Österreichs zum 70. Geburtstag des Kaisers. (Sonder-Abdruck.)

241. Adresse der Wiener Universität an die Czernowitzer zur Feier des 25jährigen. Bestehens.

\section{1}

242. Jahresbericht über Erscheinungen der Ethik aus den Jahren 1897/98. (A. f. Ph. Bd. 7, H. 2, 3.)

243.B S. Eck, „D. Fr. Strauß“. (Euphorion Bd. 8 H. 2 S. 434.)

244. B. von Carneri. (N. fr. Pr., 2. Nov.)

245. Verzeichnis von Werken aus dem Gebiete der Philosophie f. Volksbibliotheken.

246. Das sittliche Leben. [B H. Schwarz, .,Ethik“6.] (N. fr. Pr., 30. Nov.) 1902

247. Lehrbuch der Psychologie. 2. Aufl. (Am Titelblatt: 1903.)

*248. Gedanken über Reformkatholizismus. Frankfurt, N. Fr. Verlag.

*249. Zur Interpretation Spinozas. Festschr. z. 70. Geb. v. Th. Gomperz. Wien, bei Braumüller. 
*250. Uber Bedeutung und Aufgaben der Ästhetik in der Gegenwart. Antrittsvorlesung a. d. 'Techn. Hochschule. (N. fr. Pr., 12. u. 20. April.)

*251. W. Wundt zum 70. Geb. (N. fr. Pr., 19. Aug.)

252. Eine ncue Grundlegung des Idealismus. [Cohen, „Reine Logik“.] (N. fr. Pr., 19. Okt.)

\section{3}

253.B Ratzenhofer, „Ethik“. (N. fr. Pr., 8. März.)

254. Das Mädchen-Gymnasium. (N. fr. Pr., 22. März.)

*255. Eine neue Gesamt-Ausgabe der Werke L. Feuerbachs. (N. fr. Pr., 7. Juni.)

*256. Rousseau im Lichte der Pathologie. [Nach Möbius]. (N. fr. Pr., 15. Nov.)

257. Zu Roseggers 60. Geb. (Festblatt z. Nr. 207 d. Österr. Volkszeitung, 30. Juli.)

*258. Papsttum und Wissenschaft im 19.Jahrhundert. [Zum Tode Leo XIII.] (Zeit, 21. Juli.)

259. Otto Weininger. (Festschr. d. N. Wr. Journals, 25. Okt.)

*260. Göttliche Weltordnung und religionslose Sittlichkeit. [Schneider.] (Freies Wort, 1. April, Jahrg. 3 Nr. 1.)

\section{4}

261. Ethik. Geschichtlicher Abriß bis zur Gegenwart. (Neue Bearbeitung f. d. 2. Aufl. von Reins „Enzyklopädischem Handbuch der Pädagogik“".)

*262. Gedanken über Architektur. (Wiener Türmer, Almanach, hrsg. vom Akad. Architektenverein ,,Konkurrenzklub“.)

*263. Kant. Zur 100. Wiederkehr seines Todestages. (N. fr. Pr., 12. Feb.)

264. Ludwig Feuerbach. Klassiker d. Philosophie. Stuttgart bei Fromann.

*265. Ludwig Feuerbach. Zur Erinnerung an seinem 100. Geb. (Freies Wort, 1. Aug.; N. fr. Pr., 28. Juli; Das Wissen für Alle Nr. 30.)

266. Neuere Literatur zur Ästhetik. I. (Ö. R., 7. u. 18.)

*267. Der Begriff des Zufalls. Seine theoretische und praktische Bedeutung. (Gerichtssaal Bd. 64 Nr. 6.)

268.B Goldscheid, „Die Ethil des Gesamtwillens“. (Zeit, 21. Juni.)

269. Schopenhauer und Nietzsche. (N. fr. Pr., 18. Dez.)

\section{5}

*270. Zwei Schillerreden. Wien, Akad. Verlag.

*271. Kant und der Monismus. (Freies Wort, 1. April.)

*272. Schiller und die Gegenwart. (Freies Wort, 1. Mai.)

*273. Kulturmüdigkeit. (N. fr. Pr., 11. März.)

274. Künstler, Kunstschreiber und Ästhetiker. (N. fr. Pr., 16. Juli.)

275. Hermann Cohens, „Ethik des reinen Willens“. (N. fr. Pr., Sept.)

*276. Ernst Mach und seine neueste Arbeit „Erkenntnis und Irrtum". (N.fr.Pr., 24. Nov.)

*277. Schiller und die Religion. (Zeit, 23. April.)

*278. Das Nietzsche-Problem. (Ö. R., 11. Mai, Bd. III H. 28.)

*279. Krieg und Frieden. (Österr. Volkszeitung, 11. Juni.) 
280.B Mary Calkins, „An Introduction to Psychology. (Zeitschr.f.Psychologie, Okt.)

281. Geschichte der Ethik. Bd. I. 2. Aufl. Stuttgart bei Cotta.

*282. Radikaler Reformkatholizismus. [E. Jung.] (Das Freie Wort, Jahrg. 6 Nr. 14.)

283. Österreich und der Parlamentarismus. Eine Pfingstbetrachtung. (Österr. Volksztg., 3. Juni.)

\section{7}

*284. Freskomalerei in Oesterreich. (Ö. R. Bd. X H. 3, 1. Feb.)

*285. Festrede zur Feier des 20 jährigen Bestehens des Wiener VolksbildungsVereins. (Verlag d. Vereins).

*286. Rede gegen die Eroberung der Universitäten. (N. fr. Pr., 26. Nov.)

\section{8}

287. Lehrbuch der Psychologie. 3. Aufl. Stuttgart bei Cotta.

288. L. Feuerbach. (Menschheitsziele, herausg. v. Molenaar, H. 1 u. 2.)

*289. D. F. Strauß zum 100. Geb. (Jugend Nr. 4.)

*290. Ursprung und Entwicklung der Moralbegriffe. [Westermarck.] (Das Wissen für Alle Jahrg. 8 Nr. 1.)

*291. Kirche und Wissenschaft - Religion und Schule. (Freie Schule Jahrg. 3 Nr. 1.)

292. Der Fall Wahrmund. (Das freie Wort Jahrg. 8 Nr. 2.)

293. Andreas Groll und die Freskomalerei in Österreich. (Kunst und Kunsthandwerk Jahrg. XI H. 8 u. 9.)

294. Neuere Literatur zur Ästhetik. (Ö. R., Bd. 17 H. 3, 1. Nov.)

295. Adresse der Universität an den Kaiser zum 2. Dez. 1908.

296. Herder als Philosoph. (N. fr. Pr., 22. Nov.)

*297. Der Klerikalismus und die Universitäten. Der österr. Hochschulkampf im Sommer 1908. Innsbruck bei A. Edlinger.

298. Österreich und die geistige Freiheit. (Österr. Volkszeitung, 2. Dez.)

*299. Zur religiösen Zeitlage. (Freies Wort, Jahrg. VIII, 18, 15. Dez.)

*300. Wahrnehmung und Vorstellung. (Bericht des I. Intern. Kongresses f. Psychiatrie, Neurologie und Psychologie zu Amsterdam, S. 583.)

\section{9}

*301. Was heißt Bildung? (Wissen für Alle, Jänner; Sonderdruck, Wien bei H. Heller.)

*302. Rede zur Feier des 40jährigen Jubiläums des Reichsvolksschul-Gesetzes. (Erscheint im Sammelband zum erstenmal im Druck.)

303. Die Heranbildung der Lehramtskandidaten für philosophische Propädeutik. (In Beratung des Vereins für Mittelschulen, Wien.)

*304. Wissenschaft und Religion. (V Frankfurt.) Frankfurt, N. fr. Verlag. (Nachgedruckt i. Freidenker, Milwaukee, 7. März.) 
*305. Darwins Bedeutung für die Philosophie. (N. fr. Pr., 12. Feb.)

306. Marianne Hainisch als Freundin und Helferin der Volksbildung. (In F'estschr. z. 70. Geb., 25. März.)

1910

307. Warum beteilige ich mich an der Volksbildungsarbeit? (Volksbildung Nr. 8.)

308. Was uns not tut. (Neues Leben Jahrg. V Nr. 1, 1. Juli.)

*309. Denk- und Glaubensfreiheit. Anläßlich des Buches: „Liberté de conscience et liberté de science" von Luzzatti. (N. fr. Pr., 6. Aug.)

310.B Seligmann, „Kunst und Künstler von gestern und heute". (N. fr. Pr., 2. Nor.)

311. D. Hume, „Anfänge und Entwicklung der Religion“. Deutsch von W. Bolin. (DLZ., April.)

*312. Die Kirchenaustrittsbewegung und was aus ihr folgt. (Freies Wort, Jahrg. X Nr. 8, 2. Juliheft.)

\section{1}

*313. Max Stirner und L. Feuerbach. (Ö. R. Bd. 26 H. 6.)

*314. Trennung von Staat und Kirche. (10. Flugblatt d. deutschen Bundes f. weltl. Schule u. Moralunterricht.)

*315. Aus der Werkstätte der Philosophie. (V Wien.) Wien bei H. Heller.

316. Festrede zur Eröffnung des Volksbildungshauses. (MWV., Jahrg. IV 2, Febr.)

*317. Zufall, Gesetzmäßigkeit, Zweckmäßigkeit. Akademische Festrede. (Alm. d. k. Akademie der Wissenschaften.)

318. Der Monismus und die Kulturprobleme der Gegenwart. Rede auf dem Hamburger Monistenkongreß. Leipzig bei Kröner.

\section{2}

319. Geschichte der Ethik. Bd. II. 2. Aufl.

*320. B. Carneri. (Biogr. Jahrb. u. deutscher Nekrolog, Bd. 14.)

*321. Einleitung zu Rousseaus Briefe an Christophe de Beaumont, Erzbischof von Paris. (Bibliothek der Aufklärung, Frankfurt a. M., N. Fr. Verlag.)

322. Rede zur 25. Jahresfeier des Wiener Volksbildungsvereins. (MWV.)

*323. Der Kampf zwischen Glauben und Wissen in der Gegenwart. (Das monistische Jahrhundert Nr. 3.)

324. Laurenz Müllner. (Rektoratsbericht, Wien 1912/13.)

1913

*325. Zum Problem des Moralunterrichts. [Gegen Pastor Schnackenberg.] (Nordwestdeutsche Zeitg., Beitrag z. Nr. 5 u. 6; N. fr. Pr., Dez. 1912.)

326. Die Satiren des Herrn Maschine von Bergmann. (N. fr. Pr., 16. März.)

327. Zur Geschichte der Naturphilosophie [C. Siegel]. (N.fr. Pr., 21. Juni.)

328. Ewiges Leben nach Feuerbach von Wilh. Bolin. (Monist. Jahrh., 25. Mai.) 
329. David Hume und sein neuester Darsteller. (V. Schr. f. wiss. Philos., Juli.)

330. Zur Frage der Kinderkommunion. (Freie Schule.)

*331. Geist und Gedächtnis. (Pester Lloyd, 11. Mai.)

*332. 1813-2013. Ein Programm. (In Freie deutsche Jugend, Jena bei E. Diederichs.)

*333. Ostwald als Philosoph. (In Festschrift d. österr. Mo.o.-Bundes zu seinem 60. Geburtstage.)

\section{4}

*334. Gerhart Hauptmanns ,Fe:tspiel“. Ein Gespräch. („Dęr Greif“ Jahrg. 1 H. 5, Febr.)

335. Vom wahren und vom falschen Idealismus. A. Kröner, Leipzig.

336. „Vom Lebenswege“. Sammlung von Aufsätzen und Vorträgen. (Erscheint 1914. Stuttgart bei Cott9.)

337. Lehrbuch der Psychologie. 4. Aufl. (Erscheint 1914, Stuttgart bei Cotta.)

338. Feuerbach. 2. Aufl. (Erscheint 1914, Stuttgart bei Frommann.)

339. „Kritik des Idealismus". (Ein nachgelassenes Werk, erscheint 1915. Leipzig, Akad. Veragsanstalt.) 